\title{
Design and control of a novel hydraulically/pneumatically actuated robotic system for MRI-guided neurosurgery
}

\author{
${ }^{1}$ Cyrus Raoufi, ${ }^{2}$ Andrew A. Goldenberg $\&{ }^{3}$ Walter Kucharczyk
}

${ }^{1}$ Department of Applied Technology, California State University, Humboldt. ${ }^{2}$ Department of Mechanical and Industrial Engineering, University of Toronto, Toronto, Canada. ${ }^{3}$ Department of Medical Imaging, University of Toronto, Toronto, Canada. Correspondence should be addressed to Cyrus Raoufi (raoufi@humboldt.edu),Andrew A. Goldenberg (golden@mie.utoronto.ca), and Walter Kucharczyk (w.kucharczyk@utoronto.ca).

\begin{abstract}
In this paper the design of a novel modular hydraulic/pneumatic actuated tele-robotic system and a new infrastructure for MRI-guided intervention for closed-bore MRI-guided neurosurgery are presented. Candidate neurosurgical procedures enabled by this system would include thermal ablation, radiofrequency ablation, deep brain stimulators, and targeted drug delivery. The major focus is the application of the designed MR-compatible robotic system to MRIguided brain biopsy. Navigation and operating modules were designed to undertake the alignment and advancement of the surgical needle respectively. The mechanical design and control paradigm are reported.
\end{abstract}

Keywords: MR-compatible robot; Tele-surgery; Tele-robotics; Medical robot

\section{INTRODUCTION}

The common requirement for most neurosurgical procedures is to manipulate a surgical tool relative to an anatomic target. This includes aligning, orienting, and advancing the tool to a specific anatomic target in the brain. The advantages of robotic-based neu ros urgi cal procedures are well recognized in the clinical and technical community due to both the locating accuracy and the tele-surgery potential of the robotic systems. A neurosurgical procedure is a highly interactive process and the goal of neurosurgical robotic system is to provide the neurosurgeon with a reliable tool that augments his or her ability during the operation. Any surgical robotic system has to meet specific design considerations for its intended use such as safety, capability of being sterilized, fault-tolerancy, accuracy, stability, and dexterity. MRI-guided applications impose additional demands such as remote control, reduce d size, lightweight structure, and ability to operate in the MRI bore. Primarily, there is the issue of
MR-compatibility of materials and devices. Conventional robotic systems are not suitable for use inside the MRI scanner because they contain ferromagnetic materials and electrical circuits. These components cause spatial distortions and impart noise to the MR images, while conversely the magnetic field of the MRI system interferes with the electrical circuits. The strong magnetic field dictates that only nonferromagnetic materials can be used for the mechanical parts.

The major shortcoming in the use of conventional MRI systems for neurosurgery is their reliance on preoperative MR images. As surgery progresses and anatomic tissue are removed or distorted, the intracranial anatomic positional relationship of the brain and surrounding structures change. This is commonly referred to as "brain shift". Intra-operative changes due to tumor resection, brain swelling, and cere bro spinal fluid (CSF) leakage further increase brain shift $[1,2$, $3]$. As these processes are unavoidable in most neurosurgical procedures, they decrease the accuracy in all surgery that is based on preoperative MR images [3]. These intra-operative changes make it difficult or impossible to accurately determine the true intra-operative anatomic position of the anatomic target based on the preoperative images. Accurate localization during surgery thus requires the acquisition of intra-operative images. In recent years, advances in computer technology, robotics, and a significant increase in the accuracy of imaging have helped the clinicians in planning and executing surgical procedures in MRI environments. The advantages of surgical robotics are well known in clinical environments due to their precisions, accuracy, repeatability, and capability for tele-surgery [4].

In the area of MRI-guided tele-surgery, there are currently several systems under development. Tajima et al. [5] designed and built a prototype of an MRIcompatible manipulator for treatment and diagnosis of heart diseases. Larson et al. [6] developed a device to perform minimally invasive interventions in the breast with real time MRI guidance for the 
early detection and treatment of breast cancer. Engineering Services Inc. (Ontario, Canada) has also developed an MR-compatibletele-robotic system for prostate surgery [7]. Krieger et al. [8] designed and developed a novel remotely actuated manipulator (APT-MRI) to access prostate tissue under MRI guidance. Fischer et al [9] designed a robotic ass is tant system using pneumatic components aimed to be used for prostate needle placement in a closed-bore MRI scanner. Kim et al [10] designed and developed a new master-slave MR-compatible surgical manipulator for minimally invasive liver surgery. Chinzei et al. [11] designed and developed a novel MR-compatible manipulator used to position and direct an axisymmetric tool such as laser pointer or a biopsy catheter. Moser et al. [12] designed and developed a one DOF MR-compatible master-slave robotics system and a haptic interface using hydraulic transmission. Koseki et al. [13] designed and developed an endoscope manipulator for trans-nasal neurosurgery capable of being used inside the gantry of vertical field open MRI. Flueckiger et al. [14] proposed a haptic interface compatible with MR scanner for neuroscience studies. Miyata et al. [15] designed and developed an MR-compatible forceps manipulator using a new cam mechanism for the multi-function micromanipulator system for neurosurgery procedures. Engineering Services Inc. has also developed an MR-compatibletele-robotic system using water hydraulic and pneumatic actuators for neurosurgery [7]. The Calgary Health Region and University of Calgary are developing the world's first image guided neurosurgical robot (NeuroArm ${ }^{\mathrm{TM}}$ ) in collaboration with MD Robotics for micro-neurosurgery. The robot is under design and construction stage now [16].
Nakamura et al. [17] developed and manufactured the 6 DOF manipulator using non ferromagnetic materials (aluminum) and actuated by ultrasonic motors.

The goal of our research project is to design, fabricate, and test a hydraulic/pneumatic actuated MRcompatibletele-robotic system for MRI-guided neurosurgery, in particular, the brain biopsy. The mechanical design and related infrastructure are reported.

\section{ROBOT DESIGN}

\subsection{MR-compatible robotic system infra- structure}

MRI-guided tele-robotic system requires surgical planning, MR-image acquisition, human-machine interface, navigation, and sensing. To address those components required for MRI-guided intervention, an infrastructure is needed regardless of the type of surgical operation. A schematic diagram of the proposed infrastructure is illustrated in Figure 1. The entire system consists of three main subsystems as follows: (i) operating unit; (ii) power/control unit; and (iii) surgeon-machine interface unit. The operating and surgeon-machine interface units are communicating through MR images and related information using an image processing device. The image processing device is used to provide information required by both the surgeon-machine interface unit and power/control unit. The operating unit and power/control unit are communicating through power transmission and sensory information systems. Also, the surgeon-machine interface and power/control units are communicating through operation inputs created by operator input device (master).

As shown, all three units communicate through

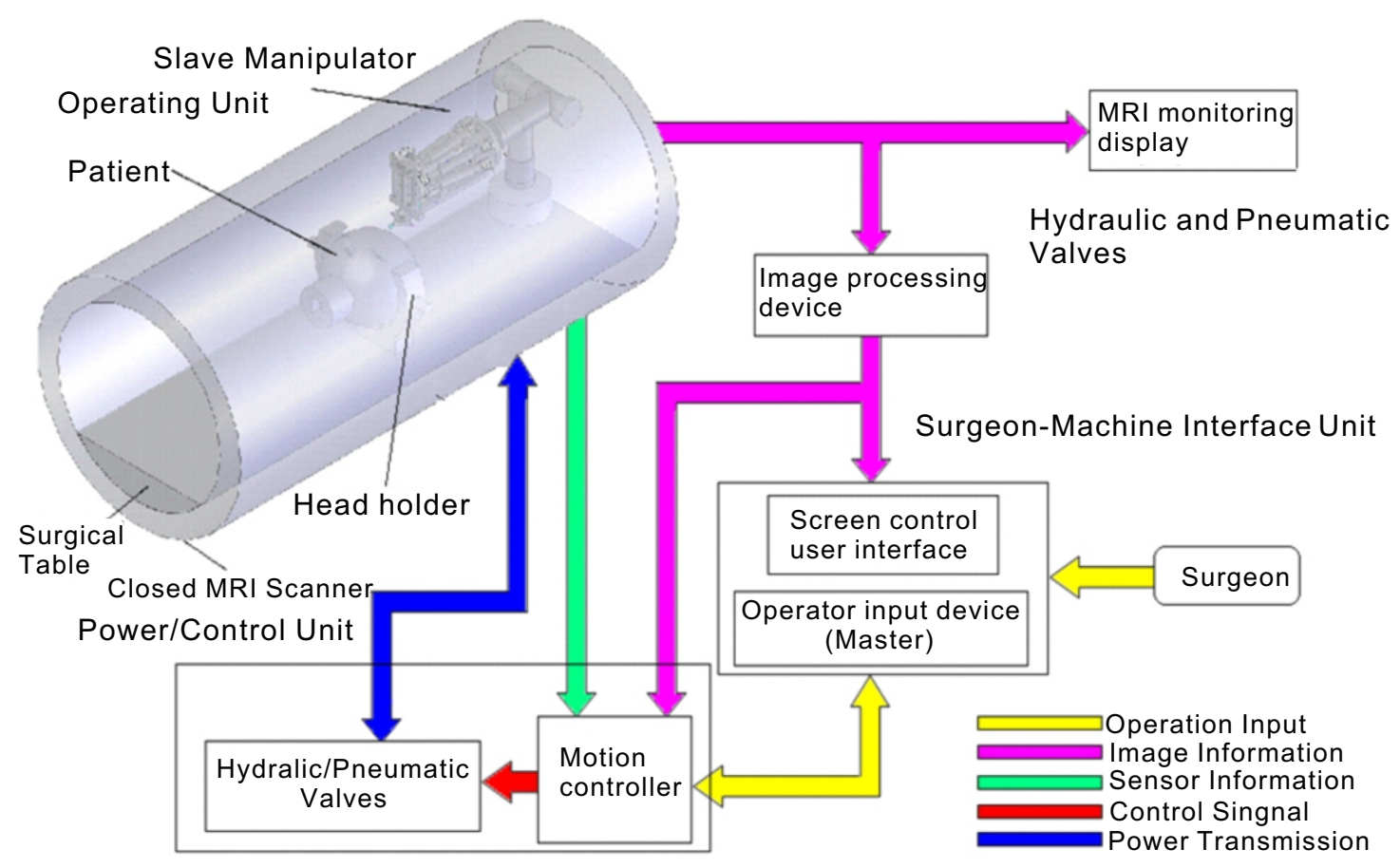

Figure 1. A schematic of the entire system. 
image information, sensory information, control signals, and power transmission. As illustrated, the visualization of the surgical tool and the target as well as surgical planning based on intra-operative MR images are completed on a display monitor in front of the surgeon in the surgeon-machine interface unit. One should note that the proposed infrastructure is based on a fundamental principle which is both the surgeon and power/control unit share the control of the tele-robotic system such that the surgeon will use his/her judgment and expertise to control the entire procedure. In other words, it is almost impossible to eliminate the surgeon from the control system and have the entire tele-robotic system performed the required task autonomously.

\subsection{Operating unit}

Operating unit comprises the slave manipulator, head holder, surgical table, and MRI scanner located in MR operating room. The patient's head and the slave manipulator are fixed to the surgical table in order to avoid any relative displacement during the surgical operation. The patient's head needs to be secured and fixed in all surgical operations to avoid unexpected motions caused by disorderly reaction of the patient's body.

Due to the presence of strong magnetic field and switching gradients both the head holder and the slave manipulator are required to beconstructed from MR-compatible materials and devices. The slave manipulator must perform the required tasks in a confined space between the patient and the bore of the MR scanner. Therefore, the slave manipulator is needed to be designed in a very compact size. In addition, the slave manipulator required to be registered with respect to the MR scanner such that the position and orientation of the surgical tool with respect to the target could be determined based on data obtained from the MR images. One should note that the patient's head must be secured during the operation as the desired position and orientation of the surgical tool with respect to the target will be obtained while the surgicaldevice is outside the patient's skull. Thus, the head holder is considered as a major component in the proposed infrastructure for application of the tele-robotic system in MR-guided neurosurgery procedures.

\subsection{Manipulator power/control unit}

The manipulator power/control unit is located in an adjacent control room at a proper distance away from the MR scanner due to electrical/electronic devices and circuits as well as non-MR-compatiblematerials used in its structure. The major function of the manipulator power/control unit is to provide required power to the slave manipulator. The power/control unit consists of two major sub-units: (i) hydraulic power units, hydraulic valves, and pneumatic valves; and (ii) motion controller devices such as computer and electrical/electronic components and circuits. The surgeon could manipulate the slave manipulator inside the MR scanner through a master manipulator located in the surgeon-machine interface unit. The motion controller in the power/control unit is also communicating with the master manipulator in the surgeon-machine interface unit to provide appropriate control signals to hydraulic and pneumatic valves. The motion controller also receives the sensory data feedback from the slave manipulator. In addition, the motion controller is also provided with the MR images data originated from the image processing device as shown in Figure 1.

\subsection{Surgeon-machine interface unit}

The major function of the surgeon-machine interface unit is to provide an interface between the entire telerobotic system and the surgeon as the end user. The goal of using tele-robotic system for MR-guided neurosurgery is not to replace the surgeon with the robot, but to provide him/her with advanced tools for remote execution of neurosurgical procedures. The unit is located in the adjacent control room to avoid magnetic interference due to use of electrical devices and non-MRI-compatible materials used in its structure. A master and a screen control user interface are the major subsystems of this unit. The images of the

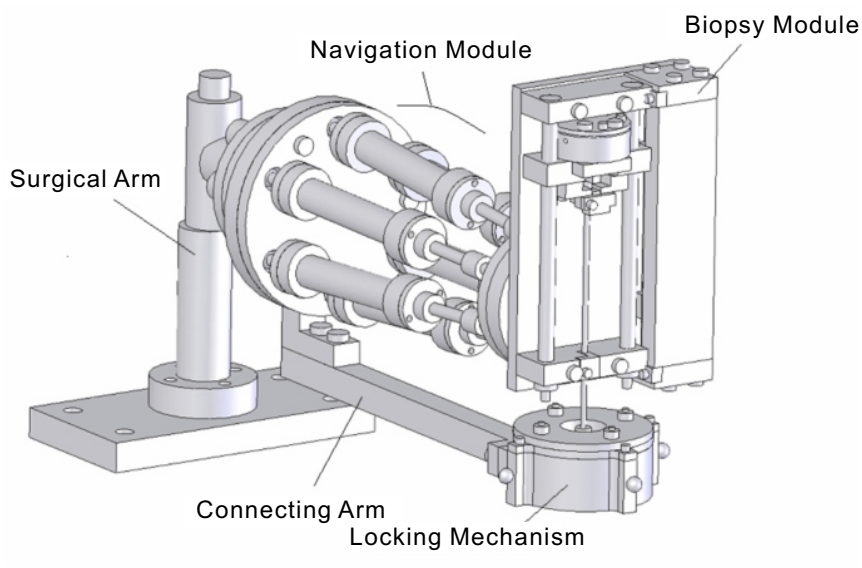

Figure 2. 3D model of the slave manipulator.

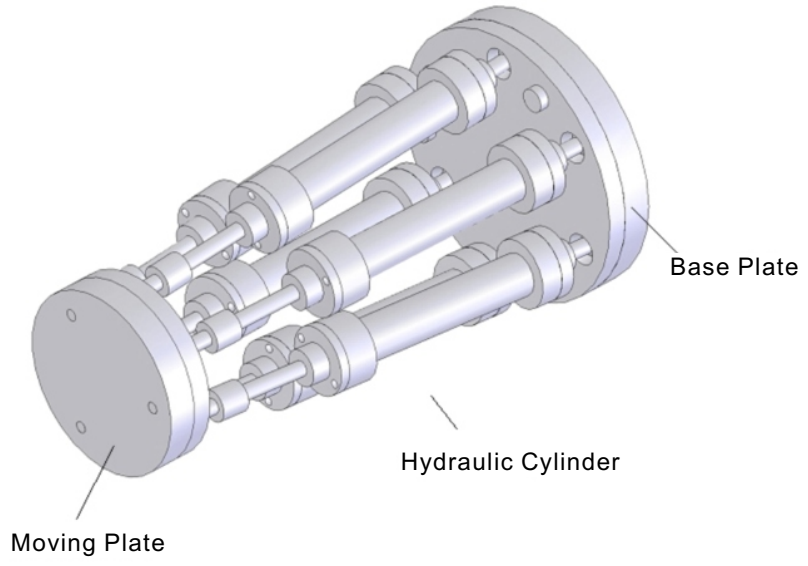

Figure 3. 3D model of the navigation module. 


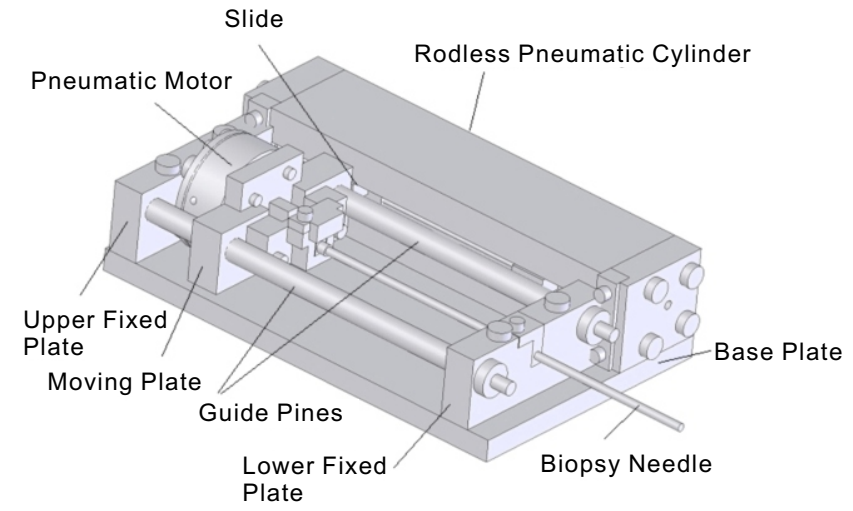

Figure 4. 3D model of the biopsy module.

slave and surrounding environment are projected on the screen to allow visualization of the target and surgical tools movements. The surgeon would manipulate the position and orientation of the surgical devices via the master controller. Surgeons strongly rely on the visual MR images as they are only reliable source of information during the operation. The screen control user interface is the unit that provides the visualization of the tissue and surgical tool while the operation progresses. There are several important challenging issues that one must consider in designing the screen control user interface including [18]: (i) integration of navigation and display with robot systems; (ii) updating the MR images in real time; (iii) providing the surgeon with means of controlling the information displayed; and (iv) finding ways to communicate useful information without overwhelming the surgeon by pointless details. The master manipulator is the unit with which surgeons could communicate their control commands. Any commonly used interfaces for human-machine interactions such as mice, joystick, touch screens, push buttons, and foot switches could be used.

\subsection{Mechanical design for the slave manipu- lator}

A 3D model of the slave manipulator is shown in Figure 2. The surgical needle is held and advanced by the biopsy module. The biopsy module is attached to the navigation module.

The navigation module is a six degrees of freedom parallel mechanism consisting of a base and a plat form interconnected through 6 legs (or struts). Six linear hydraulic actuators are used to provide required linear displacement for each leg. A locking mechanism is used to guide the needle as well as lock the robot at desired orientation. It is fixed to the base of the parallel mechanism through a connecting arm by screws. All three units (the navigation module, biopsy module, and the locking mechanism) are held by a surgical arm. The surgical arm is attached to a surgical table through a set of screws.

\subsection{Navigation module}

A 3D model of the navigation module is shown in Fig-

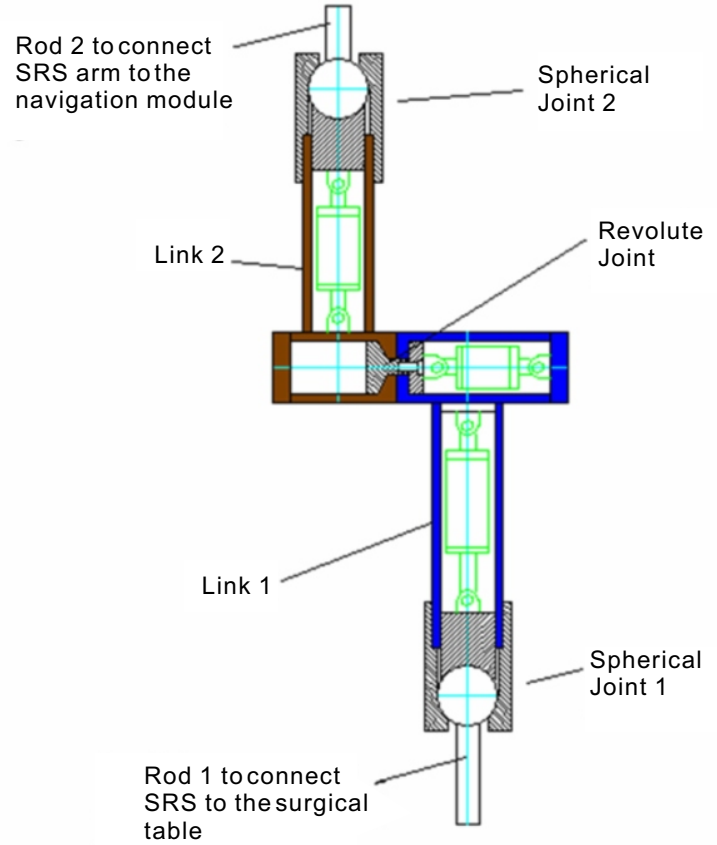

Figure 5. A schematic diagram of the surgical arm.

ure 3. It consists of a base plate and a moving plate interconnected through 6 links. Each link consists of a hydraulic linear actuator, a spherical joint, and a universal joint.

\subsection{Biopsy module and locking mechanism}

A 3D model of the biopsy module is presented in Figure 4. It is basically a three-plate mechanism including: (i) a lower fixed plate, (ii) anupper fixed plate, and (iii) a moving plate. Both lower and upper fixed plates are attached to the base plate by two sets of screws. Two guide pins are used to support the moving plate. The moving plate is moved up and down using a pneumatic rodless cylinder. The moving plate is attached to the slide of the pneumatic cylinder. A 3D model of the locking system is shown in Figure 2. The locking system consists of a connecting arm and locking mechanism. As shown, the locking mechanism is attached to the base plate of the parallel mechanism through the connecting arm. All mechanical parts are constructed from MR-compatible materials.

\subsection{Surgical arm}

The surgical arm supports both the navigation and biopsy modules during the operation. The surgical arm has to be easily maneuvered by the clinician to be located at the entry point on the patient's skull. The design of the surgical arm is shown in Figure 5. It consists of two links and three joints as follows: (i) a spherical joint 1; (ii) a revolute joint 2; and (iii) a spherical joint 2. The Spherical-Revolute-Spherical (SRS) arm is illustrated in fully deployed configuration in order to show its components and corresponding function of each component. As shown, rod 1 connects the SRS arm to the surgical table and rod 2, at the other end, connects the navigation module to the 


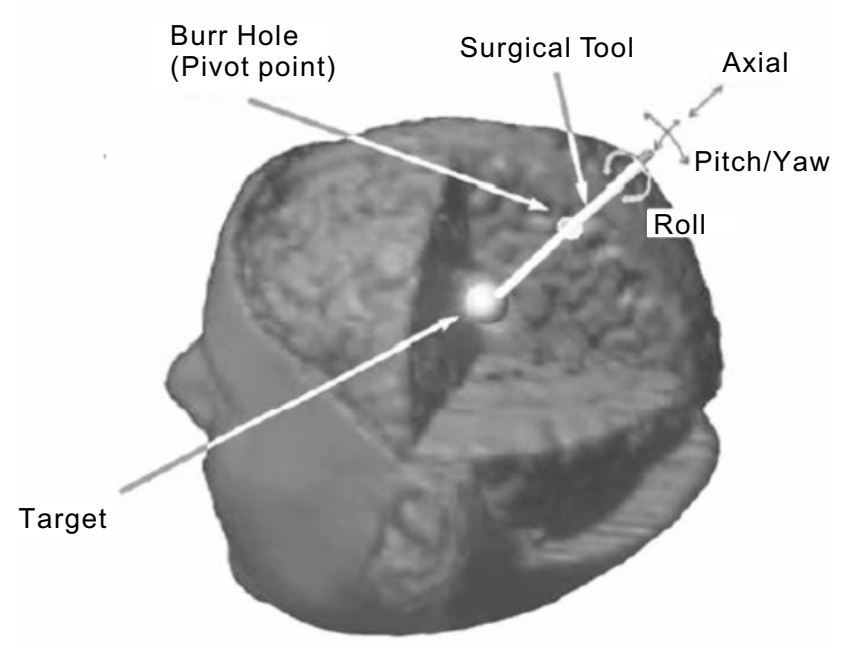

Figure 6. The target, entry point, and the needle.

SRS arm.

\section{ROBOT CONTROL}

\subsection{Needle alignment}

An entry point, a surgical tool and a target are depicted in Figure 6. Required motions to align and advance the surgical tool with respect to the target are also shown. The surgical tool is rotated about the burr-hole by Yaw and Pitch angles. This point is also called the pivot point. The conventional surgical tool placement at an entry point includes the following three tasks: (i) move the needle tip to the entry point using 3 DOFs; (ii) orient the needle by pivoting around the entry point using 2DOF (Yaw and Pitch angles); and (iii) insert the needle into the body using 1 DOF (translation along a straight trajectory). Using the proposed tele-robotic system shown in Figure 1, the brain biopsy procedure would be carried out as follows:

(1) Preoperative imaging stage. The patient is placed inside the MRI scanner and preoperative images are obtained.

(2)Surgical planning stage. Based on the preoperative images, an entry point is determined and the incision is made by a surgeon.

(3)Pre-alignment stage. The slave manipulator is attached to the surgical table, and the navigation module and biopsy needle are manually located at the entry point. Although this stage doesn't require high accuracy in positioning, the slave has to be locked such that the surgical tool is positioned at the entry point. Accurate alignment with respect to target will be done in the next stage;

(4)Real time navigation stage. The patient is moved into the bore of MRI scanner. The navigation module is maneuvered remotely in order to align the surgical tool with the desired direction based on intraoperative images.

(5)Intra-operative operation stage. The operation is carried out by advancing the needle using intra- operative images as visual feedback. When the needle reaches the target, it is rotated by 180 degrees in order to cut the tissue specimen (tumor). Then the needle is pulled out completing the operation.

(6)Final stage. The MRI table is moved out the MRI bore. The slave manipulator and head holder are detached from the table and patient's skull respectively.

\subsection{Robot control architecture}

As mentioned, the surgeon adjusts the orientation of the surgical tool (yaw and pitch angles) based on visual MR images through the master. The inverse kinematics of the navigation module is used to obtain the desired length of each strut related to the desired position and orientation of the needle biopsy.

The hydraulic/pneumatic circuit of the system and overall control system are shown in Figure 7 and Figure 8 respectively. Six MR-compatible hydraulic cylinders are equipped with six fiber optic encoders to feedback the actual length of each strut. Using inverse kinematic of the navigation module, the desired length of each strut of the navigation module is determined. A PID controller provides a control signal that drives a hydraulic proportional valve in each servo control loop. The hydraulic valve controls the length of the strut by regulating the flow from/to each hydraulic actuator. In addition, a pneumatic valve (V7) is used to control the tip position of the biopsy needle. The semi-rotary pneumatic motor is also actuated by an on/off pneumatic valve (V8).

A block diagram of the control algorithm used in the controller is shown in Figure 9. The inputs are six feedback displacement signals from the slave side (LA1, LA2, LA3, LA4, LA5, and LA6), two signals form master side including desired Yaw and Pitch angels, and desired length of each strut (LD1, LD2, LD3, LD4, LD5, and LD6). The outputs are control signals ( $\mathrm{S} 1, \mathrm{~S} 2, \mathrm{~S} 3, \mathrm{~S} 4, \mathrm{~S} 5$, and $\mathrm{S} 6)$ to control the proportional valves.

A PC-based supervisory controller is designed to control entire system as illustrated in Figure 10. The trajectory of each joint is calculated based on the inverse kinematics in a PC-based supervisory controller and fed to each joint controller RS485 Bus. As shown in Figure 10, six optical encoders are used to feedback the position signals to six microprocessors. Each actuator has individual microprocessor to control its proportional valve.

\section{CONCLUSION AND FUTURE WORK}

We have designed an MR-compatible tele-robotic system that can be used for orientation and advancement of a biopsy needle on the brain biopsy procedure. The robot has been designed such that it will perform desired tasks inside MR scanner GE Signa 1.5T. To date, design and analysis of the entire system have been completed. Material selection and the controller architecture and its component have been finalized. A physical prototype of the slave manipulator is in the process of being constructed. Current and future 


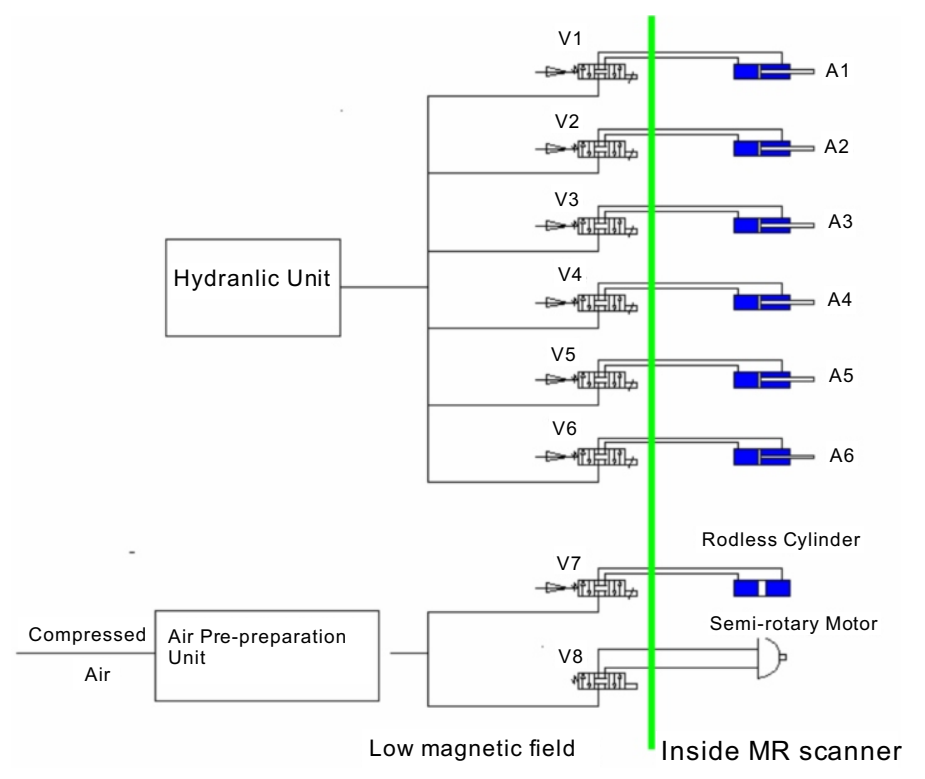

Figure 7. Aschematic of hydraulic/pneumatic circuit.

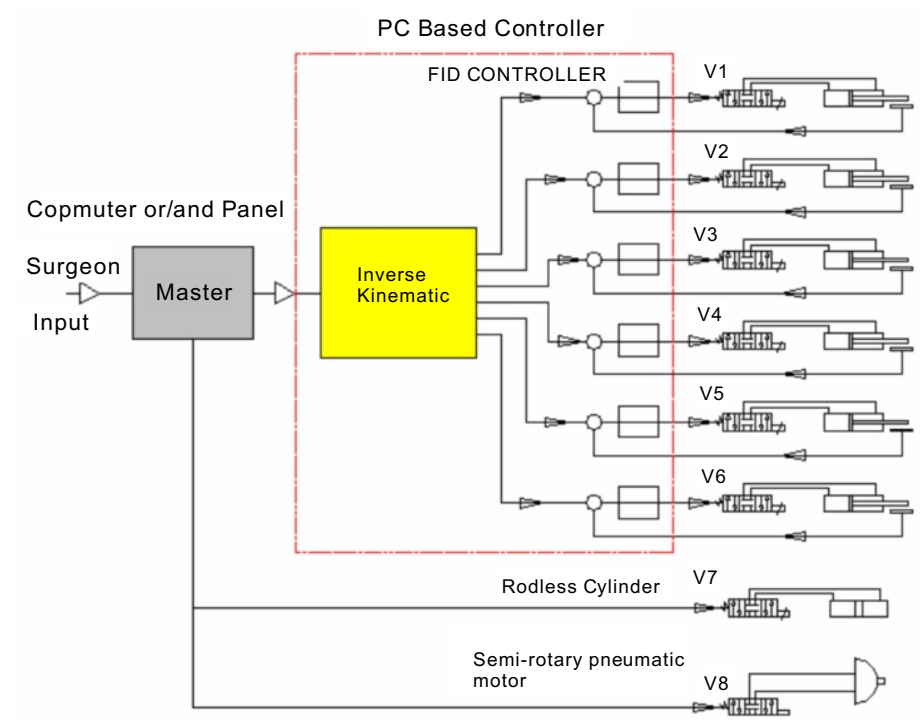

Figure 8. Aschematic of overall control architecture.

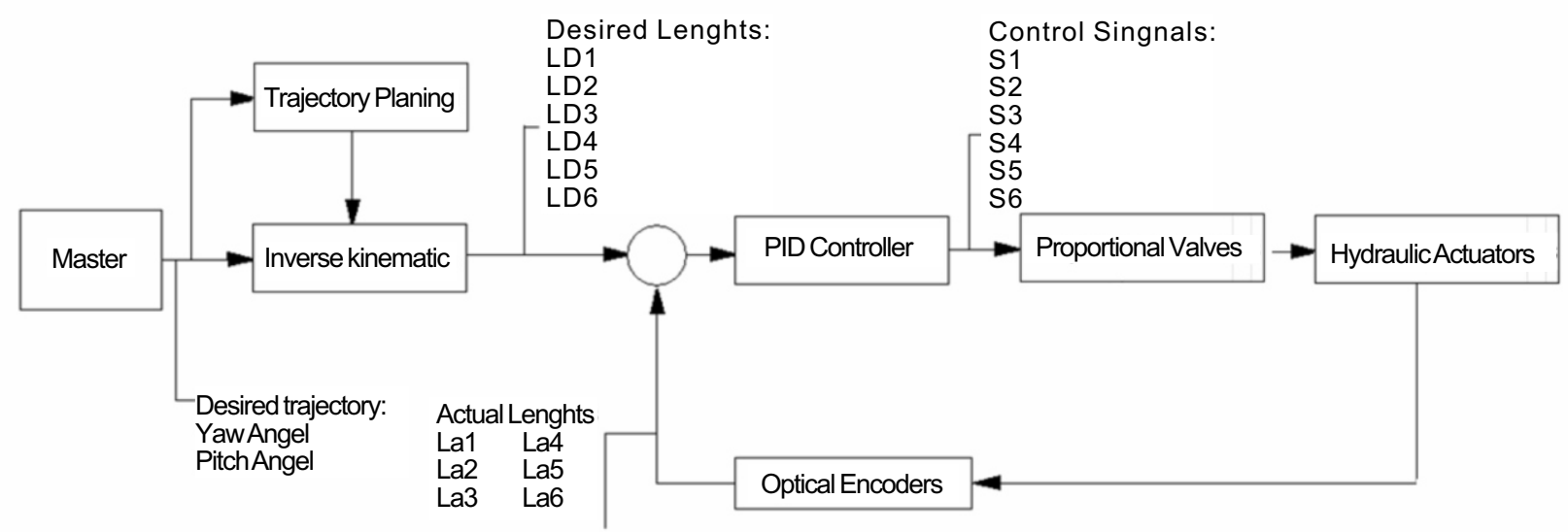

Figure 9. Aschematic of overall control architecture. 


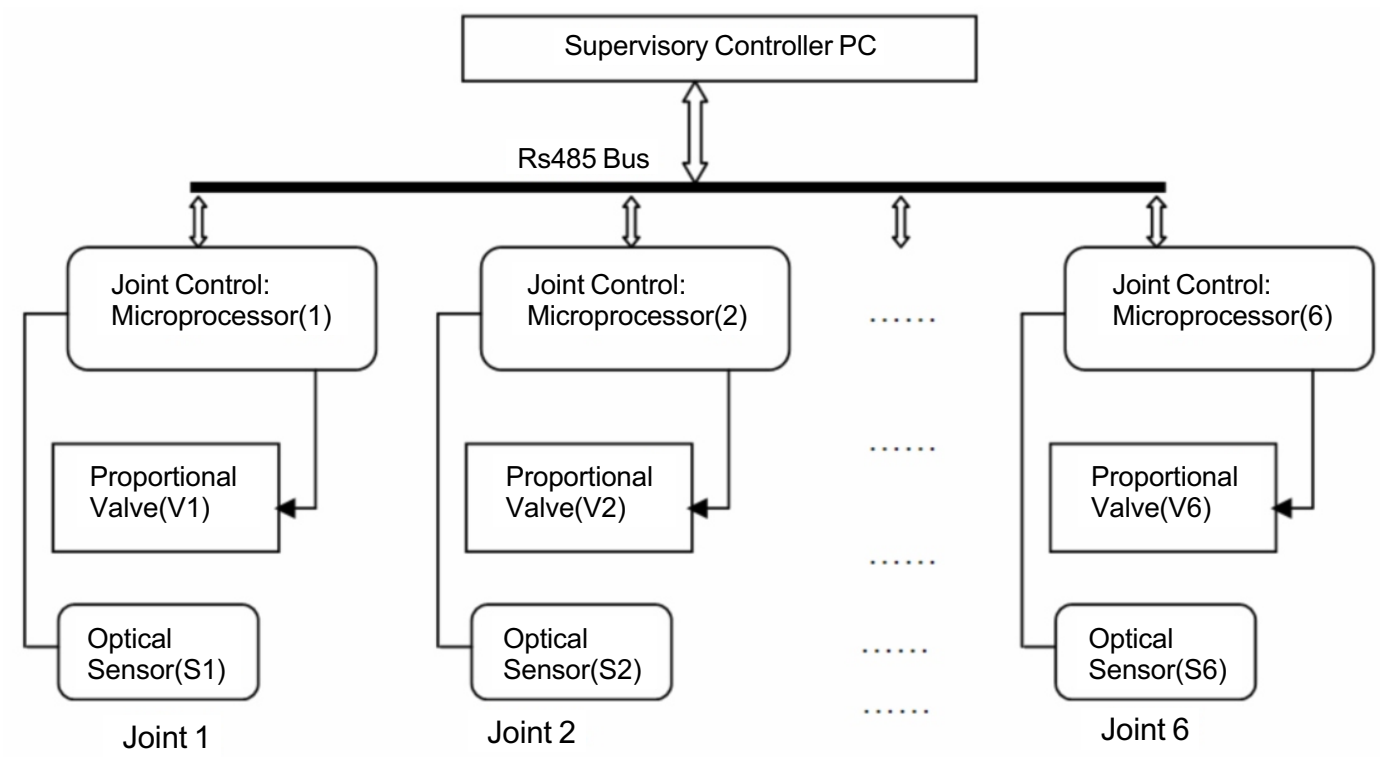

Figure 10. Supervisory control configuration.

work includes the development of the slave manipulator and performance of series of experimental tests inside the MR scanner using the first physical prototype.

\section{ACKNOWLEDGEMENTS}

This work was partially supported by Natural Sciences and Engineering Research Council of Canada (NSERC), grant held by Professor Andrew A. Goldenberg and Ontario Research and Development Challenge Fund (ORDCF), grant held by Professor W. Kucharczyk. The authors would like to thank Engineering Services Inc., Canada, and MRI department in Toronto General Hospital, Canada, for providing help and equipment.

\section{REFERENCE}

[1]R. D. Howe, \& Y. Matsuoka. Robotics for Surgery. Annual Review of Biomedical Engineering 1999, 1:211-240.

[2]J. Kettenbach, D. F. Kacher, S.K. Koskinen, S. Silverman, A. Nabavi, D. Geringt, C. Tempany, R. B. Schwartz, R.Kikinis, P. K. Black \& F.A. Jolesz. Interventional and Intra-operative Magnetic Resonance Imaging. Annual Review Biomedical Engineering 2000, 2:661-90.

[3]A. Nabavi, D. F. Kacher, D. T. Gering et al. Neurosurgical procedure in 0.5 Tesla, open-configuration intraoperative MRI planning, visualization, and navigation. Automedia 2001, 00:135 .

[4]K. Chinzei, N. Hata, F.A. Jolesz, \& R. Kikinis, Medical Image Computing and Computer-Assisted Intervention - MICCAI 2000. Third International Conference Proceedings 2000, pages 921-30.

[5]F. Tajima, K. Kishi, K. Nishizawa, K. Kan, Y. Nemoto, H. Takeda et al. Development of MR-compatibleSurgical Manipulator toward a Unified Support System for Diagnosis and Treatment of Heart Disease. Proc .of MICCA O2 2002, pages 83-90.

[6]B. Larson, N. Tsekos \& A. g. Erdman. A Robotic Device for Minimally Invasive Breast Intervention with Real-Time MRI Guidance. Proc. Of the Third IEEE Symposium on Bioinformatics and Bioengineering 2003.

[7]Engineering Services Inc., http://www.esit.com, Internal report.

[8]A. Krieger, R. Susil, C. Menard, J. Coleman, G. Fichtinger, E. Atalar, \& L. Whitcomb. Design of a Novel MRI Compatible
Manipulator for Image Guided Prostate Intervention. IEEE Trans. On Biomedical Engineering 2005, 52(2):306-313.

[9]G.S. Fischer, I. Iordachita, S. P. DiMaio \& G. Fichtiger. Design of a Robot for Transperineal Prostate Needle Placement in MRI scanner. IEEE International Conference on Mechatronics 2006, page 6.

[10]K. Daeyoung et al. A New, Compact MR-Compatible Surgical Manipulator for Minimally Invasive Liver Surgery. MICCAI 2002, pages 99-106.

[11]K. Chinzei \& K. Miller. MR Guided Surgical Robot. Proc. 2001 Australian Conference on Robotics and Automation 2001, Sydney.

[12]R. Moser, R. Gassert, E. Burdet, L. Sache, H. Woodtli, J. Erni, W. Maeder \& H. Bleuler. An MR-compatibleRobot Technology. Proc. Of the IEEE, International Conference on Robotics \& Automation 2003.

[13]Y. Koseki, T. Washio, K. Chinzei \& H. Iseki. Endoscope Manipulator for Trans-nasal Neurosurgery, Optimized for and Compatible to Vertical Field Open MRI. Proc. of MICCAI 2002, pages 114-121.

[14]M. Flueckiger, M. M. Bullo et al. FMRI compatible haptic interface actuated with traveling wave ultrasonic motor. IAS Annual Meeting (IEEE Industry Applications Society) 2005, 3:2075-2082.

[15]N. Miyata , E. Kobayashi, D. Kim, K. Masamue et al. Micrograsping Forceps Manipulator for MR-Guided Neurosurgery. MICCAI 2002, pages 107-113.

[16]Calgary HealthTrust, www.cbi.ucalgary.ca/CHT, 2004.

[17]R. Nakamura, K. Masamune, Y. Nishikawa, E. Koboayashi, I. Sakuma,T. Dohi, H. Iseki \& K. Takakura. Development of a sterilizable MRI-compatible manipulator for stereotactic neurosurgery. Proc. Of Computer Assisted Radio Surgery (CAR'99) 1999.

[18]R. Taylor \& D. Stoianovici. Medical Robotics in ComputerIntegrated Surgery. IEEE Transaction on Robotics and Automation 2003, 32(5):765-781. 